\title{
Epithelial Cell-Specific MyD88 Signaling Mediates Ischemia/ Reperfusion-induced Intestinal Injury Independent of Microbial Status
}

\author{
Marcus Mühlbauer, MD, PhD*, Ernesto Perez-Chanona, $\mathbf{M S}^{\star}, \dagger$, and Christian Jobin, PhD* ${ }^{\star} \dagger$ \\ *Departments of Medicine, Microbiology and Immunology, and Pharmacology, University of North \\ Carolina, Chapel Hill, North Carolina \\ †Department of Medicine and Department of Infectious Diseases \& Pathology, University of \\ Florida, Gainesville, Florida.
}

\section{Abstract}

The Toll-like receptor/MyD88 signaling pathway has been shown to mediate protective functions during intestinal exposure to various noxious events. The goal of this study was to define the role of bacteria and MyD88 signaling in intestinal response to damage using an ischemia-reperfusion (I/R)-induced injury model. We showed that conventionalized mice displayed a better outcome to I/R-induced injury than germ-free mice $(3.8 \pm 1.98$ vs. $11.8 \pm 1.83, P<0.05)$. However, mice with intestinal epithelial cell (IEC)-specific deletion of $M y d 88$ ( $M y d 88^{\mathrm{IEC}-/-}$ ) were protected from I/Rinduced injury compared with $M y d 88^{\mathrm{f} / \mathrm{f}}$ control mice. $M y d 88^{\mathrm{IEC}-} /-$ mice also displayed a significantly reduced bacterial translocation ( $85 \%$ ) into lymph nodes compared with $M y d 88^{\mathrm{f} / \mathrm{f}}$ mice. Expression of $\underline{\mathrm{ccl} 2}$ and $\mathrm{cxcll}$ mRNA was significantly reduced ( $85 \%$ and $62 \%$, respectively) in intestinal tissue of $M y d 88^{\mathrm{IEC}-/-}$ mice compared with $M y d 88^{\mathrm{f} / \mathrm{f}}$ mice, which associated with a reduced number of myeloperoxidase-positive cells in intestinal tissues of I/R-exposed Myd $88^{\mathrm{IEC}-1-}$ mice. Immunohistochemistry analysis showed a reduced $\mathrm{IgA}$ deposition and complement staining in ischemic tissue of $M y d 88^{\mathrm{IEC}-/-}$ mice compared with $M y d 88^{\mathrm{f} / \mathrm{f}}$ mice. These findings suggest that I/R-induced intestinal injury involves IEC-derived MyD88 signaling leading to increased IgA deposition/degradation, and complement activation in conjunction with an influx of neutrophils mediated by chemokine production.

\section{Keywords}

ischemia; reperfusion; MyD88; intestinal epithelial cells

Copyright @ 2013 Crohn's \& Colitis Foundation of America, Inc.

Reprints: Christian Jobin, PhD, Department of Medicine, University of Florida, Gainesville, FL 32608 (christian.jobin@medicine.ufl.edu)..

Supplemental digital content is available for this article. Direct URL citations appear in the printed text and are provided in the HTML and PDF versions of this article on the journal's Web site (www.ibdjournal.org).

The authors have no conflicts of interest to disclose.

Author Contribution: MM and CJ conceived and designed the experiments; MM and EP-C performed the experiments; $\mathrm{MM}$ and $\mathrm{CJ}$ analyzed the data; $\mathrm{MM}$ and $\mathrm{CJ}$ wrote the article. 
The intestinal epithelium constitutes a crucial physical barrier separating the host from the luminal compartment containing various noxious agents such as bacteria, bacterial products, and food-derived particles. ${ }^{1-3}$ In addition to its barrier function, the intestinal epithelium responds to numerous bacterial stimuli through the action of various conserved innate sensors such as Toll-like receptors (TLRs). ${ }^{4}$ In the case of commensal bacteria, this dialogue between the microbiota and intestinal epithelial cells (IECs) is important for the maintenance of intestinal homeostasis through the induction of antimicrobial peptides, toning of the innate and adaptive immune responses, and the fortification of the barrier. ${ }^{5} \mathrm{~A}$ compromised intestinal barrier could have deleterious consequences for the host as luminal contents may gain access to the mucosal immune system and enter systemic circulation, triggering a damaging immune cell response. The epithelium is constantly facing injury caused by various luminal irritants, but these episodes rarely lead to life-threatening situations because the host has developed an efficient adaptive wound-healing response to maintain homeostasis. ${ }^{6}$ However, extreme challenges such as hypoxic, ischemic, or radiation events often overpower the wound-healing response, thereby exposing the host to damaging luminal products. For example, intestinal ischemia can lead to sepsis through bacterial translocation. ${ }^{7}$ Intestinal ischemia-reperfusion (I/R) injury can occur under several clinical conditions like sepsis, hemorrhage, mesenteric ischemia due to blood clots, neonatal necrotizing enterocolitis, and even small bowel transplantation. ${ }^{8}$ Reperfusion after ischemic events causes epithelial cell death and disruption of barrier function leading to invasion of bacteria. ${ }^{9}$ Antibiotic use is the mainstay of treatment to prevent sepsis from bacterial translocation after intestinal ischemic events and has even been shown to be protective before the inciting event. ${ }^{10}$

Interestingly, the microbiota seems to play a protective role in various experimental injury models including dextran sodium sulfate and radiation. ${ }^{11-13}$ In contrast, global deletion of myeloid differentiation primary response gene (Myd88), an adaptor protein in innate immune signaling, protects mice from I/R-induced injury. ${ }^{14}$ Moreover, in a model of necrotizing enterocolitis in newborn mice, TLR/MyD88-mediated intestinal injury seems to be controlled by nucleotide-binding oligomerization domain-2 signaling. ${ }^{15}$ However, the role of microbes in injury response to $\mathrm{I} / \mathrm{R}$ is still unclear because most studies linking bacteria to I/R response have used reduction strategies (antibiotic treatment) and/or innate sensor-deficient mice. Numerous cells, including IEC and mucosal immune cells, express MyD88, and the cell type specific function of this molecule in I/R-mediated injury response is unknown.

In this study, we investigated the impact of the microbiota using germ-free and conventionalized mice and defined the role of IEC-specific MyD88 signaling in I/R-induced intestinal injury. We report that IEC-derived MyD88 signaling promotes I/R-induced injury. This deleterious effect is likely mediated by 2 main events: (1) IEC-dependent MyD88 signaling leading to increased amount of luminal $\operatorname{IgA}$ and subsequent complement activation during the ischemic phase and (2) IEC-MyD88-dependent expression of neutrophil chemoattractants in IECs, further damaging intestinal tissue. 


\section{MATERIALS AND METHODS}

\section{Generation of $M y d 88^{\mid E C}-/-$ Mice}

All mice were on a C57BL/6 background. Villin-Cre transgenic mice were crossed to $M y d 88^{\mathrm{f} / \mathrm{f}}$ mice (a generous gift from Dr Anthony DeFranco, UCSF, CA), which contain loxP sites flanking exon 3 to generate mice lacking exon 3 of $M y d 88$ gene in IECs $\left(M y d 88^{\mathrm{IEC}-/-}\right) .{ }^{16}$ Tail snips were collected from all pups generated from crosses, and genomic DNA was isolated using a Qiagen Blood and Tissue Kit (Qiagen; Valencia, CA). Primers used for genotyping were as followed: Villin-Cre promoter (5'GCGGTCTGGCAGTAAAAACTATC-3', and 5'GTGAAACAGCATTGCTGTCACTT-3') and MyD88 ${ }^{\mathrm{f} / \mathrm{f}}\left(5^{\prime}-\right.$ GTTGTGTGTGTCCGACCGT-3' and 5'-GTCAGAAACAACCACCACCATGC-3').

For phenotypic analysis, RNA was isolated from IEC and splenocytes as previously described, ${ }^{17}$ and expression of MyD88 was determined by PCR. Isolation of IECs was performed as previously described. ${ }^{18}$ Amplicons were resolved on a 2\% agarose gel and visualized using a Kodak Gel Logic Imager 200 series (Kodak; Rochester, NY). Myd88 primers used were as followed: 5'-TTGGATGCCTGGCAGGGG-3' and 5'-

TCCTTCTTCATCGCCTTG-3'. The amplicon length including exon 3 was 565 bp and the amplicon length without exon 3 was $384 \mathrm{bp}$. Deletion of exon 3 in epithelial cells was also confirmed by sequencing of the PCR products. Purity of IEC and splenocytes isolation was confirmed by amplifying the IEC-specific gene product Villin and the immune cell-specific marker (neutrophils, monocytes, and B-cell subtypes) CD11b using the respective set of primers 5'-CCCCCATCTTCCAACAAT-3', 5' -GGACCTGAAATAGCCTCGTAG-3' (amplicon length: $546 \mathrm{bp}$ ); and 5'-GGCGCTGTCTACATTTTTTAT-3' and 5'GCTCCCCAACCAGTGTATAAT-3' (amplicon length 574 bp) (Fig, Supplemental Digital Content 1, http://links.lww.com/IBD/A334). Low levels of CD11b contamination that occurred during IEC isolation account for the wild-type $M y D 88$ bands that appear in IECspecific Myd88 knockout samples of small intestine and colon tissue.

\section{I/R-induced Injury}

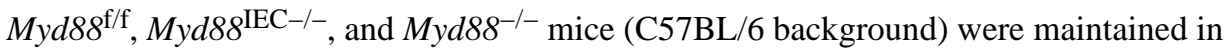
standard housing cages in specific pathogen free-conditions. To study the impact of the microbiota on I/R injury, 1 cohort of germ-free (GF) wild-type mice (C57BL/6 background) was transferred to specific pathogen-free conditions for 4 weeks, and another cohort was kept in GF conditions. For all experiments, mice $(\mathrm{n}=3-5)$ were anesthetized under $1 \%$ isofluorane, supplemented by $10 \mathrm{mg} / \mathrm{kg}$ ketamine, and $0.1 \mathrm{mg} / \mathrm{kg}$ buprenorphine, both injected subcutaneously. A mid-line laparotomy was made, and peripheral branches of the superior mesenteric artery were occluded with aneurysm clips with a force of $50 \mathrm{~g}$ (Kent Scientific, Torrington, $\mathrm{CN}$ ), to create a 3- to 5-cm region of ischemic ileum adjacent to the cecum. Collateral blood flow through the intestine was blocked using aneurysm clips across the intestine and collateral vessels, demarking the region of ischemic intestine. Hematoxylin was administered to the edges of ischemic tissue to mark them, and then the incision was closed with surgical staples. Ischemia was maintained for 60 minutes, and then the incision was reopened, the clamps were removed, and the incision was reclosed. We have optimized 
this protocol to maximize injury and maintain a low mortality rate (4\%), as previously published. ${ }^{19}$ The mice were maintained in a heated room for a variable amount of time $(0$, 1.5 , or 4 hours) without anesthesia for the reperfusion phase of injury. To account for any systemic reaction to injury, healthy tissue adjacent to injured tissue was harvested as an internal healthy control, as previously described. ${ }^{19}$ This approach was used in lieu of "sham" animals, which do not appropriately control for the systemic effects that originate from I/R-induced injury. All animal experiments were approved by the Institutional Animal Care and Use Committee of the University of North Carolina at Chapel Hill.

\section{Murine Sample Collection and Histological Evaluation}

Mice were anesthetized using isoflurane, and then killed by cervical dislocation. The colon was dissected and flushed with ice-cold phosphate-buffered saline, longitudinally splayed, Swiss rolled, fixed in $10 \%$ formalin for 24 hours, and then embedded in paraffin. Damage severity was evaluated using Hematoxylin and eosin-stained sections by an investigator blinded to the experimental conditions. The scoring system is based on an IEC apoptosis/ necrosis system, where a score of 1 signified a loss of only the villus tips; a score of 2 corresponded to loss of $50 \%$ of the villus; a score of 3 indicated a loss of the entire villus, but with maintenance of the crypt; and a score of 4 signified complete loss of the epithelial layer. ${ }^{20}$ The ischemic tissue was divided into 4 quarters, a score was given to each quarter separately and then added to generate a final damage score.

\section{Immunohistochemistry and Immunofluorescence}

Immunohistochemistry (IHC) staining was performed according to the manufacturer's specifications, as previously described. ${ }^{17}$ Primary antibodies and dilutions were as followed: myeloperoxidase, 1:400 (Neomarkers, Fremont, CA), PIgR, 1:50 (R\&D Systems; Minneapolis, MN), IgA, 1:1000 (R\&D Systems; Minneapolis, MN), and complement 3d, 1:50 (R\&D Systems; Minneapolis, MN). IHC for activated caspase 3, 1:400 (R\&D Systems; Minneapolis, MN) was performed as described. ${ }^{21}$ All sections were counterstained with hematoxylin and eosin.

\section{RNA Isolation and Real-time PCR}

RNA isolation from ileal tissues and subsequent cDNA amplification and analysis were performed as previously described. ${ }^{17}$ Specificity and linearity of amplification for each primer set were determined by melting curve analysis and calculation of the slope from serial diluted samples. Relative fold changes were determined using the $\Delta \Delta \mathrm{CT}$ calculation method. Values were normalized to the internal control GAPDH or $\beta$-actin. Primers:

GAPDH (5'-GGTGAAGGTCGGAGTCAACGGA-3' and 5'-

GAGGGATCTCGCTCCTGGAAGA-3'), $\beta$-actin (5'-

TGGAATCCTGTGGCATCCATGAAAC-3' and 5' -

TAAAACGCAGCTCAGTAACAGTCCG-3'), cxcll (5' -

GCTGGGATTCACCTCAAGAA-3' and 5' - TCTCCGTTACTTGGGGACAC-3'), ccl2 (5'GCTGCTACTCATTCACTGGCAA-3' and 5' - TGCTGCTGGTGATTCTCTTGTA-3'), and $\operatorname{tnf}\left(5^{\prime}\right.$-ATGAGCACAGAAAGCATGATC-3' and 5'-

TACAGGCTTGTCACTCGAATT-3’). 


\section{Bacterial Translocation and Culture}

For all bacterial translocation assays, mice were subjected to 60 minutes of ischemia followed by 4 hours of reperfusion. Mice were killed, and 2 mesenteric lymph nodes adjacent to the damaged tissue were harvested and weighed. The homogenates were lysed in $1 \mathrm{~mL}$ phosphate-buffered saline using a Mini BeadBeater-8 (BioSpec, Bartlesville, OK) and plated on sheep brain heart infusion plates (Remel, Lenexa, KA) and incubated in aerobic and anaerobic conditions for 24 hours, at which time colonies were counted.

\section{Statistical Analyses}

Unless specifically noted, statistical analyses were performed using GraphPad Prism version 5.0a (GraphPad, La Jolla, CA). Comparisons of mouse studies were made with nonparametric analysis of variance, and then a Mann-Whitney $U$ test. All graphs depict mean \pm SEM. Experiments were considered statistically significant if $P<0.05$.

\section{RESULTS}

\section{Commensal Microbes Alleviate I/R-induced Ileal Damage}

Although wide-spectrum antibiotic treatment attenuates I/R-induced intestinal injury in mice, this experimental approach may have confounding effects on the host. ${ }^{10}$ To stringently evaluate the impact of bacteria on intestinal injury response, we used GF and conventionalized wild-type mice. Conventionalized mice and GF mice (3-5 mice per group) were exposed to either 60-minute ischemia (I) or 60-minute ischemia followed by 180 minute reperfus ion (I/R), and tissue injury was assessed by histological analysis. Although no difference was observed between the histological damage scores of the conventionalized and GF group ( $12.7 \pm 1.80$ vs. $11.6 \pm 0.70$, respectively) in the ischemic phase (Fig. 1A, B), a significant improvement was noted in conventionalized mice versus GF mice $(3.8 \pm 1.98$ vs. $11.8 \pm 1.83, P<0.05$ ) during the reperfusion phase (Fig. 1A, B). This finding suggests that bacteria may promote beneficial responses during the reperfusion phase of $\mathrm{I} / \mathrm{R}$-induced tissue injury.

\section{IEC-specific Myd88 Deletion Alleviates I/R-induced Injury}

To assess early stages of epithelial cell damage and recovery during $\mathrm{I} / \mathrm{R}$, the reperfusion time was reduced from 180 minutes to 90 minutes for the subsequent experiments. We have previously optimized I/R-induced injury to maximize epithelial damage. ${ }^{19}$ To define the role of bacteria in I/R-mediated injury, we selectively deleted Myd 88 in IEC by crossing $M y d 88^{\mathrm{f} / \mathrm{f}}$ mice to Villin-Cre mice to generate $M y d 88^{\mathrm{IEC}-/-}$ mice. The extent of ischemiainduced intestinal injury was similar in $M y d 88^{\mathrm{f} / \mathrm{f}}$ and $M y d 88^{\mathrm{IEC}-/-}$ mice $(8.8 \pm 0.86$ vs. 10.8 \pm 1.16 ; not significant) during the ischemic phase (Fig. 2A). However, after 90 minutes of reperfusion, $M y d 88^{\mathrm{IEC}-/-}$ mice showed greater epithelial restitution compared with $M y d 88^{\mathrm{f} / \mathrm{f}}$ mice ( $4.6 \pm 1.29$ vs. $10 \pm 1.67$, respectively; $P<0.05$ ) (Fig. $2 \mathrm{~B}$ ). The protective recovery observed in $M y d 88^{\mathrm{IEC}-/-}$ mice was similar to that of $M y d 88^{-/-}$mice $(4.6 \pm 1.29$ vs. $4 \pm 1.27$, respectively) suggesting that MyD88 signaling from the IEC compartment was mainly responsible for the phenotype. 
Since defective IEC-derived MyD88 alleviated epithelial cell damage, we predicted that Myd $88^{\mathrm{IEC}-/-}$ mice should have a diminished translocation of bacteria. As expected, a significant decrease of colony-forming units in lymph nodes was observed in I/R-exposed Myd $88^{\mathrm{IEC}-/-}$ mice compared with $M y d 88^{\mathrm{f} / \mathrm{f}}$ mice (67 \pm 27 vs. $452 \pm 185$, respectively; $P<$ $0.05 ;$ Myd88 $8^{-/-}: 181 \pm 121$ ) (Fig. 3).

\section{Deletion of MyD88 Signaling Reduces I/R-induced Apoptosis in Epithelial Cells}

Intestinal tissues undergo hypoxia that contributes to IEC apoptosis and epithelial injury during I/R. ${ }^{22}$ Consequently, I/R-induced IEC apoptosis was compared between $M y d 88^{\mathrm{IEC}-/-}$ and $M y d 88^{\mathrm{f} / \mathrm{f}}$ mice by evaluating active caspase-3 using IHC staining. A significant decrease in the number of caspase-3-positive cells was observed in $M y d 88^{\mathrm{IEC}-1-}$ mice compared with Myd88f/f mice (6.4 \pm 2.4 vs. $26.3 \pm 0.53$, respectively; $P<0.05$ ) (Fig. $4 \mathrm{~A}, \mathrm{~B})$, a level similar to $M y d 88^{-/-}$mice $(6.2 \pm 2.1)$. Healthy tissue rarely showed the presence of activated caspase-3 (Fig. 4A). No difference in epithelial cell proliferation was observed between the 3 genotypes as measured by Ki-67 staining (data not shown).

\section{IEC-derived MyD88 Signaling Promotes Chemokine Gene Expression and Neutrophil Recruitment in I/R-injured Tissue}

Neutrophils have been linked to enhanced tissue damage in various injury models, including $\mathrm{I} / \mathrm{R}$ in the myocardium, liver, and intestine, ${ }^{23-26}$ therefore, we next examined expression of various chemoattractant genes involved in neutrophil recruitment. A significant induction of Tnf, Cxcll, and Ccl2 mRNA expression was observed in I/R-exposed tissues of Myd88f/f mice compared with control healthy tissues $(5.146 \pm 1.802,37.32 \pm 7.119$, and $31.09 \pm$ 9.391 fold, respectively). Interestingly, expression of $\mathrm{Cxcll}$ and $\mathrm{Ccl} 2$ was not induced in the injured tissue of $M y d 88^{\mathrm{IEC}-/-}$ and $M y d 88^{-/-}$mice compared with $M y d 88^{\mathrm{f} / \mathrm{f}}$ mice (cxcll: $14.11 \pm 3.632,4.524 \pm 0.846$ vs. $37.32 \pm 7.119$, respectively; $P<0.05)$ (ccl2: $4.524 \pm 0.846$, $5.561 \pm 1.275$ vs. $31.09 \pm 9.391$, respectively; $P<0.05$ ) (Fig. 5). The expression of Tnf in injured tissue is the same across all genotypes. IHC analysis for myeloperoxidase, a marker for neutrophils, showed a $\sim 50 \%$ reduction of infiltrating myeloperoxidase-positive cells in injured tissue of $M y d 88^{\mathrm{IEC}-/-}$ mice compared with $M y d 88^{\mathrm{f} / \mathrm{f}}$ mice $(17.03 \pm 3.08$ vs. $40.13 \pm$ 2.52, respectively; $P<0.05$ ) (Fig. 6). Taken together, these findings suggest that IECderived MyD88 signaling promotes I/R-induced injury, and associates with elevated chemokine expression and neutrophil infiltration.

\section{PIgR Expression and IgA Deposition/denaturation is Reduced in I/R-injured Myd88 ${ }^{\mathrm{IEC}-/-}$ Mice}

Complement activation plays an important role in I/R-injury and inhibition of complement alleviates this pathological response. ${ }^{27}$ Hypoxia-induced denaturation of IgA has recently been shown to activate complement through the mannose-binding lectin pathway. ${ }^{28}$ Interestingly, we observed reduced expression of IgA transporter polymeric immunoglobulin receptor in healthy tissue of $M y d 88^{\mathrm{IEC}-1-}$ mice compared with $M y d 88^{\mathrm{f} / \mathrm{f}}$ mice (Fig. 7). We sought to determine the level of IgA staining in $M y d 88^{\mathrm{IEC}-/-}$ mice after 60 minutes of ischemia, a timepoint where most "neo-antigens" are uncovered. ${ }^{29}$ As expected, IHC analysis showed decreased IgA staining in ischemia-exposed Myd88 $8^{\mathrm{IEC}-l-}$ 
mice compared with $M y d 88^{\mathrm{f} / \mathrm{f}}$ mice (Fig. 8). The IgA staining pattern was predominantly observed at the tip of the villus of $M y d 88^{8 / f}$ mice, a region typically affected by hypoxic conditions, such as ischemia.

Moreover, the staining pattern for activated complement in damaged tissue paralleled the one observed with IgA (Fig. 9), suggesting that complement activation is partly dependent on the presence of denaturated IgA. Taken together, these findings indicate that MyD88 signaling contributes to I/R injury at least partly through a higher abundance of luminal IgA that has the potential to bind to neoantigens in damaged tissue, leading to complement activation during reperfusion.

\section{DISCUSSION}

TLR/Myd88 signaling plays a critical role in various intestinal injury responses, including chemical, radiation, and I/R-induced injury. ${ }^{11,13,30}$ This signaling pathway mediates host responses to bacterial colonization/infection, and depletion of intestinal commensal bacteria through antibiotic treatment attenuates I/R-induced intestinal injury. ${ }^{10}$ Thus, we hypothesized that bacteria use MyD88 signaling to mediate injury responses in intestinal epithelial cells. However, intestinal injury was exacerbated in GF mice compared with conventionalized mice suggesting that bacteria protect against injury. The central role of MyD88 in TLR2, TLR4, TLR5, and TLR9 signaling ${ }^{31}$ suggests that bacteria-mediated protective effects would likely be dependent on this signaling protein. Surprisingly, deletion of Myd88 from the intestinal epithelial compartment did not worsen I/R-induced injury but rather protected the epithelium. This finding suggests that exacerbated I/R-induced injury in GF mice is because of a Myd88-independent pathway. It is still unclear how the microbiota protect against I/R-induced intestinal damage. Numerous innate sensors have been linked to intestinal injury responses including the Nod-like receptor Nod2. ${ }^{15}$ Nod2 seems to prevent I/R-induced injury through downregulation of TLR4 expression in epithelial cells, making these cells less susceptible to damage-induced apoptosis. ${ }^{15}$ Alternatively, the microbiota may protect the epithelium independently of innate sensor signaling. Generation of bacterial-derived products such as short-chain fatty acids has been shown to help maintain intestinal homeostasis and responses to injury. ${ }^{32,33}$ Further studies would be required to identify the mechanism by which bacteria mediate protective effects after I/R exposure.

Our observation that MyD88 signaling promotes the injury response independently of bacteria is in line with previous reports studying the function of TLR $4^{30}$ and MyD88 ${ }^{14}$ in I/R-induced injury and by other groups using a related model of intestinal injury. ${ }^{15,34}$. Furthermore, we identified the epithelial compartment as the source of MyD88-induced tissue damage. Mice defective in epithelial-derived MyD88 signaling showed diminished neutro-phil infiltration, reduced binding of sIgA to neoantigens and a subsequently reduced complement activation that correlated with the histological damage.

Interestingly, other reports suggested that TLR2/MyD88 signaling protects against I/Rinduced injury. ${ }^{35}$ The contrasting role of MyD88 in intestinal injury response could be because of variations in microbial communities in mice housed in different facilities. Different microbial compositions can modulate wound-healing responses. ${ }^{36}$ 
Neutrophil depletion has been shown to alleviate damage in intestinal I/R-induced injury. ${ }^{24,25}$ Interestingly, $c c l 2$ and $c x c l 1$ mRNA expression were reduced in $M y d 88^{\mathrm{IEC}-/-}$ mice compared with control $M y d 88^{\mathrm{f} / \mathrm{f}}$ mice. This reduction correlated with a decreased infiltration of neutrophils into damaged tissue of $M y d 88^{\mathrm{IEC}-/-}$ mice compared with control $M y d 88^{\mathrm{f} / \mathrm{f}}$ mice. Multiple studies have shown a positive association between mRNA expression of $C c l 2$ and $C x c l l$ and infiltration of neutrophils into ischemic tissue. ${ }^{25,37}$ MyD88 signaling is essential for neutrophil recruitment and tissue damage in myocardial I/R-induced injury. ${ }^{23}$ Victoni et al ${ }^{14}$ also reported alleviated tissue damage in $M y d 88^{-/-}$ mice compared with wild-type mice and demonstrated reduced neutrophil recruitment to the intestine on I/R injury. Therefore, epithelial-derived MyD88 signaling could be implicated in neutrophil recruitment and tissue damage through the induction of chemokines such as Ccl2 and Cxcll.

In addition to reduced chemokine expression as seen in this study, Frantz et al ${ }^{38}$ recently reported a reduced $p I g R$ mRNA expression in $M y d 88^{\mathrm{IEC}-I-}$ mice compared with $M y d 88^{\mathrm{f} / \mathrm{f}}$ mice, leading to a decrease in levels of luminal sIgA. Mucosal antibodies have the potential to recognize "neoantigens" revealed on ischemic cells and to activate the complement pathway, leading to exacerbate damage during I/R-induced injury. ${ }^{10,30,39,40}$ Increased intestinal IgA and IgM deposition after I/R injury in mice was previously reported. ${ }^{10}$ Because $M y d 88^{\mathrm{IEC}-/-}$ mice have lower basal levels of luminal IgA, we hypothesized that these mice would display diminished deposition of $\operatorname{IgA}$ in injured tissue. We focused on IgA because a previous study reported similar levels of IgG and IgM but lower IgA levels in Myd $88^{-/-}$mice compared with wild-type mice. ${ }^{41}$ In this study, IHC analysis confirmed decreased IgA deposition in ischemic tissue from $M y d 88^{\mathrm{IEC}-/-}$ mice compared with $M y d 88^{\mathrm{f} / \mathrm{f}}$ mice. Recently denatured IgA was shown to activate complement through the mannose-binding lectin pathway and denaturation of $\operatorname{IgA}$ can be induced by the combination of acidosis and beta-galactosidases present in ischemic intestinal tissue. ${ }^{28,42}$ Importantly, inhibiting complement, specifically through the mannose-binding lectin pathway, prevents I/R-induced injury. ${ }^{27,43}$ Interestingly, both IgA and complement displayed similar deposition on injured tissue of $M y d 88^{8 / f}$ mice, a pattern reduced in $M y d 88^{\mathrm{IEC}-/-}$ mice. In contrast, GF mice have reduced $\operatorname{IgA}$ production ${ }^{44}$ and severe tissue damage, suggesting that a mechanism other than $\operatorname{IgA}$ /complement activation mediates intestinal injury in this system. For example, impaired production of cytoprotective molecules such as short-chain fatty acids in GF mice may explain their increased susceptibility to I/R injury. ${ }^{32}$

In summary, this study identified IEC-derived Myd88 signaling as promoting I/R-induced injury. We showed that IEC-derived MyD88 signaling leads to increased basal levels of luminal $\operatorname{IgA}$ and activated complement in ischemic tissue. In addition, IEC-derived MyD88 controls the induction of neutrophil chemoattractants. This dual MyD88-dependent function is likely important for I/R-induced intestinal damage. These findings indicate that IECderived MyD88 signaling should be considered a central player in I/R-induced injury and that therapeutic intervention could focus on this signaling protein.

\section{Supplementary Material}

Refer to Web version on PubMed Central for supplementary material. 


\section{Acknowledgments}

The authors thank Dr Balfour Sartor and Maureen Bower of the National Gnotobiotic Rodent Resource Center at University of North Carolina for assistance with germ-free mice (NIH P40 R018603). We thank Dr. Anthony DeFranco, University of California San Francisco, CA for the generous gift of MyD88f/f mice. Histology was performed at the CGIBD histology core (P30 DK034987). The authors thank Ms Brigitte Allard for technical assistance throughout this project and Dr Javier Guzman for editorial assistance.

Supported by National Institutes of Health grants R01DK047700 and R01DK073338 (C.J.) and Crohn's and Colitis Foundation of America (M.M.). The funders had no role in the study design, data collection and analysis, decision to publish, or preparation of the article.

\section{REFERENCES}

1. Camilleri M, Madsen K, Spiller R, et al. Intestinal barrier function in health and gastrointestinal disease. Neurogastroenterol Motil. 2012; 24:503-512. [PubMed: 22583600]

2. Hansen J, Gulati A, Sartor RB. The role of mucosal immunity and host genetics in defining intestinal commensal bacteria. Curr Opin Gastroenterol. 2010; 26:564-571. [PubMed: 20871399]

3. McDole JR, Wheeler LW, McDonald KG, et al. Goblet cells deliver luminal antigen to CD103+ dendritic cells in the small intestine. Nature. 2012; 483:345-349. [PubMed: 22422267]

4. Abreu MT. Toll-like receptor signalling in the intestinal epithelium: how bacterial recognition shapes intestinal function. Nat Rev Immunol. 2010; 10:131-144. [PubMed: 20098461]

5. Maynard CL, Elson CO, Hatton RD, et al. Reciprocal interactions of the intestinal microbiota and immune system. Nature. 2012; 489:231-241. [PubMed: 22972296]

6. Kayama H, Takeda K. Regulation of intestinal homeostasis by innate and adaptive immunity. Int Immunol. 2012; 24:673-680. [PubMed: 22962437]

7. Vollmar B, Menger MD. Intestinal ischemia/reperfusion: microcirculatory pathology and functional consequences. Langenbecks Arch Surg. 2010; 396:13-29. [PubMed: 21088974]

8. Mallick IH, Yang W, Winslet MC, et al. Ischemia-reperfusion injury of the intestine and protective strategies against injury. Dig Dis Sci. 2004; 49:1359-1377. [PubMed: 15481305]

9. Watanabe T, Kobata A, Tanigawa T, et al. Activation of the MyD88 signaling pathway inhibits ischemia-reperfusion injury in the small intestine. Am J Physiol Gastrointest Liver Physiol. 2012; 303:G324-G334. [PubMed: 22628037]

10. Yoshiya K, Lapchak PH, Thai T-H, et al. Depletion of gut commensal bacteria attenuates intestinal ischemia/reperfusion injury. Am J Physiol Gastrointest Liver Physiol. 2011; 301:1020-1030.

11. Fukata M, Michelsen KS, Eri R, et al. Toll-like receptor-4 is required for intestinal response to epithelial injury and limiting bacterial translocation in a murine model of acute colitis. Am J Physiol Gastrointest Liver Physiol. 2005; 288:G1055-G1065. [PubMed: 15826931]

12. Burdelya LG, Krivokrysenko VI, Tallant TC, et al. An agonist of toll-like receptor 5 has radioprotective activity in mouse and primate models. Science. 2008; 320:226-230. [PubMed: 18403709]

13. Rakoff-Nahoum S, Paglino J, Eslami-Varzaneh F, et al. Recognition of commensal microflora by toll-like receptors is required for intestinal homeostasis. Cell. 2004; 118:229-241. [PubMed: 15260992]

14. Victoni T, Coelho FR, Soares AL, et al. Local and remote tissue injury upon intestinal ischemia and reperfusion depends on the TLR/MyD88 signaling pathway. Med Microbiol Immunol. 2009; 199:35-42. [PubMed: 19941004]

15. Richardson WM, Sodhi CP, Russo A, et al. Nucleotide-binding oligomerization domain-2 inhibits toll-like receptor-4 signaling in the intestinal epithelium. Gastroenterology. 2010; 139:904-917. e6. [PubMed: 20580721]

16. Hou B, Reizis B, DeFranco AL. Toll-like receptors activate innate and adaptive immunity by using dendritic cell-intrinsic and -extrinsic mechanisms. Immunity. 2008; 29:272-282. [PubMed: 18656388]

17. Uronis JM, Mühlbauer M, Herfarth HH, et al. Modulation of the intestinal microbiota alters colitisassociated colorectal cancer susceptibility. PLoS One. 2009; 4:e6026. [PubMed: 19551144] 
18. Jijon HB, Madsen KL, Walker JW, et al. Serum amyloid A activates NF- $\kappa$ B and proinflammatory gene expression in human and murine intestinal epithelial cells. Eur J Immunol. 2005; 35:718726. [PubMed: 15724247]

19. Goldsmith JR, Perez-Chanona E, Yadav PN, et al. Intestinal epithelial cell-derived $\mu$-opioid signaling protects against ischemia reperfusion injury through PI3K signaling. Am J Pathol. 2013; 182:776-785. [PubMed: 23291213]

20. Jilling T, Simon D, Lu J, et al. The roles of bacteria and TLR4 in rat and murine models of necrotizing enterocolitis. J Immunol. 2006; 177:3273-3282. [PubMed: 16920968]

21. Sun X, Threadgill D, Jobin C. Campylobacter jejuni induces colitis through activation of mammalian target of rapamycin signaling. Gastroenterology. 2012; 142:86-95. e5. [PubMed: 21963787]

22. Feinman R, Deitch EA, Watkins AC, et al. HIF-1 mediates pathogenic inflammatory responses to intestinal ischemia-reperfusion injury. Am J Physiol Gastrointest Liver Physiol. 2010; 299:G833G843. [PubMed: 20689059]

23. Feng Y, Zhao H, Xu X, et al. Innate immune adaptor MyD88 mediates neutrophil recruitment and myocardial injury after ischemia-reperfusion in mice. Am J Physiol Heart Circ Physiol. 2008; 295:H1311-H1318. [PubMed: 18660455]

24. Hernandez LA, Grisham MB, Twohig B, et al. Role of neutrophils in ischemia-reperfusion-induced microvascular injury. Am J Physiol. 1987; 253:H699-H703. [PubMed: 3631303]

25. Sisley AC, Desai T, Harig JM, et al. Neutrophil depletion attenuates human intestinal reperfusion injury. J Surg Res. 1994; 57:192-196. [PubMed: 8041137]

26. Martinez-Mier G, Toledo-Pereyra LH, McDuffie JE, et al. Neutrophil depletion and chemokine response after liver ischemia and reperfusion. J Invest Surg. 2001; 14:99-107. [PubMed: 11396626]

27. Huang Y, Qiao F, Atkinson C, et al. A novel targeted inhibitor of the alternative pathway of complement and its therapeutic application in ischemia/reperfusion injury. J Immunol. 2008; 181:8068-8076. [PubMed: 19017999]

28. Terai I, Kobayashi K, Vaerman J-P, et al. Degalactosylated and/or denatured IgA, but not native IgA in any form, bind to mannose-binding lectin. J Immunol. 2006; 177:1737-1745. [PubMed: 16849483]

29. Gralinski MR, Park JL, Ozeck MA, et al. LU 51198, a highly sulfated, low-molecular-weight heparin derivative, prevents complement-mediated myocardial injury in the perfused rabbit heart. $\mathrm{J}$ Pharmacol Exp Ther. 1997; 282:554-560. [PubMed: 9262315]

30. Moses T, Wagner L, Fleming SD. TLR4-mediated Cox-2 expression increases intestinal ischemia/ reperfusion-induced damage. J Leukoc Biol. 2009; 86:971-980. [PubMed: 19564573]

31. Murray PJ, Smale ST. Restraint of inflammatory signaling by interdependent strata of negative regulatory pathways. Nat Immunol. 2012; 13:916-924. [PubMed: 22990889]

32. Baba AA, Srinivas M, Shariff A, et al. Role of short chain fatty acids in mesenteric ischemia reperfusion injury in rats. Eur J Pediatr Surg. 2010; 20:98-101. [PubMed: 20017090]

33. Tedelind S, Westberg F, Kjerrulf M, et al. Anti-inflammatory properties of the short-chain fatty acids acetate and propionate: a study with relevance to inflammatory bowel disease. World $\mathbf{J}$ Gartroenterol. 2007; 13:2826-2832.

34. Neal MD, Sodhi CP, Dyer M, et al. A critical role for TLR4 induction of autophagy in the regulation of enterocyte migration and the pathogenesis of necrotizing enterocolitis. J Immunol. 2013; 190:3541-3551. [PubMed: 23455503]

35. Aprahamian CJ, Lorenz RG, Harmon CM, et al. Toll-like receptor 2 is protective of ischemiareperfusion-mediated small-bowel injury in a murine model. Pediatr Crit Care Med. 2008; 9:105109. [PubMed: 17906593]

36. Iizuka M, Konno S. Wound healing of intestinal epithelial cells. World J Gartroenterol. 2011; 17:2161-2171.

37. Santen S, Wang Y, Laschke MW, et al. Rho-kinase signalling regulates CXC chemokine formation and leukocyte recruitment in colonic ischemiareperfusion. Int J Colorectal Dis. 2010; 25:10631070. [PubMed: 20593289] 
38. Frantz AL, Rogier EW, Weber CR, et al. Targeted deletion of MyD88 in intestinal epithelial cells results in compromised antibacterial immunity associated with downregulation of polymeric immunoglobulin receptor, mucin-2, and antibacterial peptides. Mucosal Immunol. 2012; 5:501512. [PubMed: 22491177]

39. Chen J, Crispín JC, Tedder TF, et al. B cells contribute to ischemia/reperfusion-mediated tissue injury. J Autoimmun. 2009; 32:195-200. [PubMed: 19342197]

40. Williams JP, Pechet TT, Weiser MR, et al. Intestinal reperfusion injury is mediated by IgM and complement. J Appl Physiol. 1999; 86:938-942. [PubMed: 10066708]

41. Woods A, Soulas-Sprauel P, Jaulhac B, et al. MyD88 negatively controls hypergammaglobulinemia with autoantibody production during bacterial infection. Infect Immun. 2008; 76:1657-1667. [PubMed: 18227170]

42. Asp NG, Dahlqvist A. Human small intestine $\beta$-galactosidases: specific assay of three different enzymes. Anal Biochem. 1972; 58:591-593.

43. Matthijsen RA, Derikx JPM, Steffensen R, et al. Mannose-binding lectin null alleles are associated with preserved epithelial cell integrity following intestinal ischemia reperfusion in man. Mol Immunol. 2009; 46:2244-2248. [PubMed: 19477015]

44. Hapfelmeier S, Lawson MAE, Slack E, et al. Reversible microbial colonization of germ-free mice reveals the dynamics of IgA immune responses. Science. 2010; 328:1705-1709. [PubMed: 20576892] 


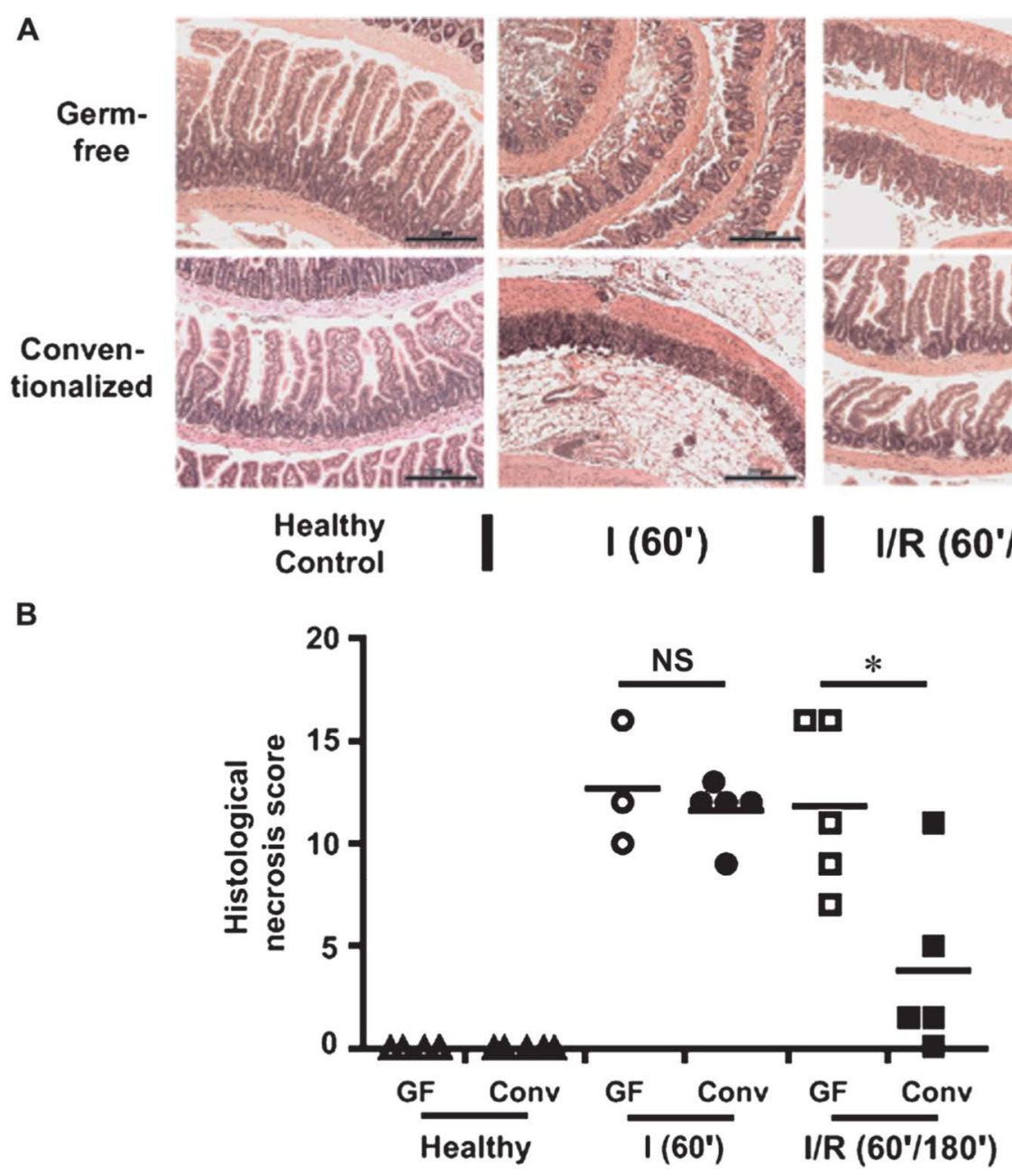

FIGURE 1.

Colonization with commensal bacteria alleviates I/R-induced injury. Cohorts of 3-5 germfree (GF) wild-type mice and 5 conventionalized (Conv) wild-type mice were subjected to either 60-minute ischemia only (I) or 60-minute ischemia followed by 180 -minute reperfusion $(\mathrm{I} / \mathrm{R})$. Scale bars $=200 \mu \mathrm{m}$. A, Representative images of hematoxylin and eosinstained ileal sections are shown. B, Histological intestinal damage scores are depicted as mean $\pm \mathrm{SEM} ; * P<0.05$. NS, not significant. 

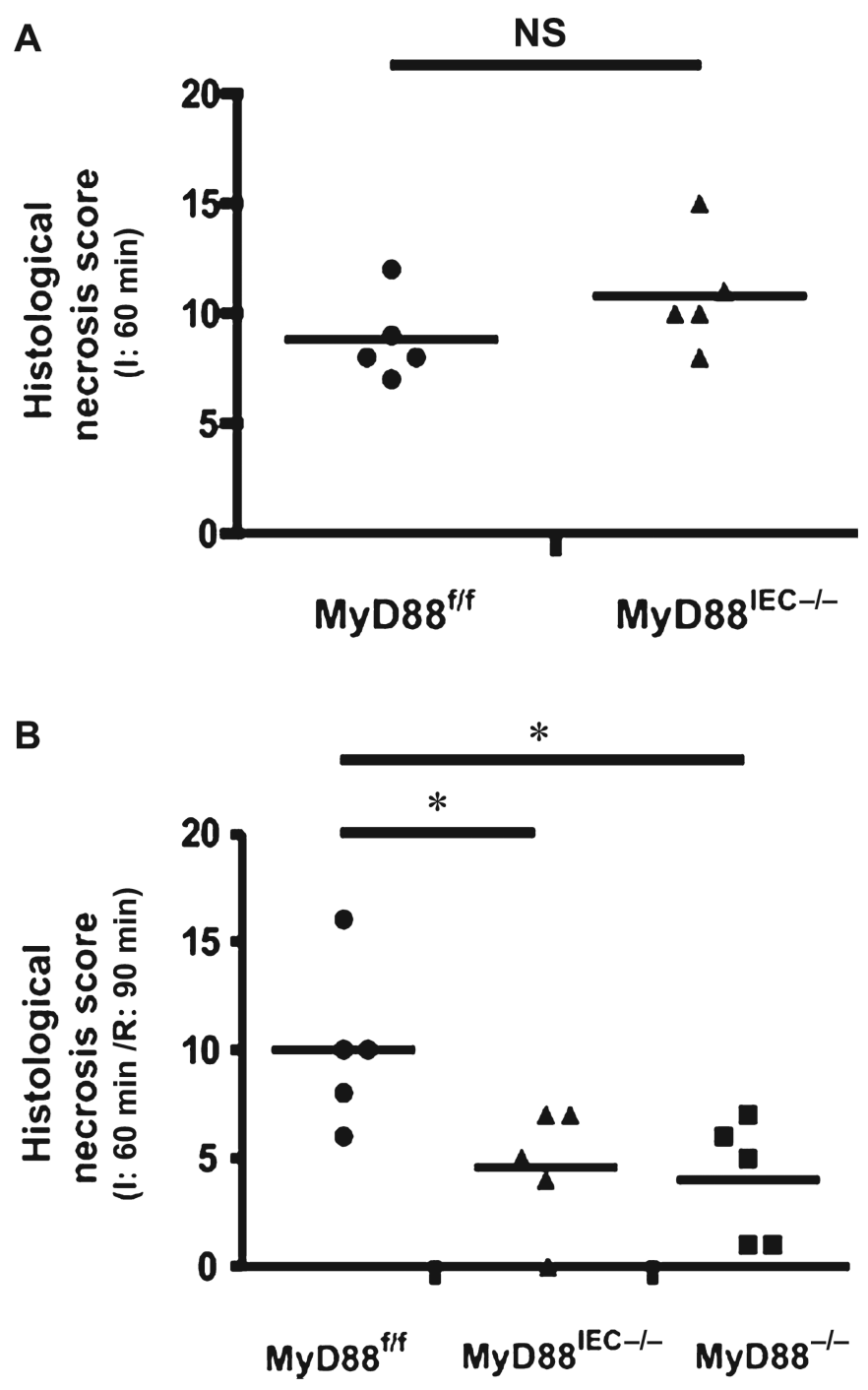

FIGURE 2.

IEC-specific Myd88 deletion protects from I/R injury. Myd88 $8^{\mathrm{f} / \mathrm{f}}, M y d 88^{\mathrm{IEC}-/-}$, and $M y d 88^{-/-}$ mice $(n=5)$ were subjected to 60-minute ischemia (I) only (A) or 60-minute ischemia followed by 90 -minute reperfusion (I/R) (B). Histological damage scores were evaluated on damaged ileal tissues using a modified scoring system. All graphs depict mean $\pm \mathrm{SEM}$; ${ }^{*} P<$ 0.05. Results are representative of 2 independent experiments. NS, not significant. 


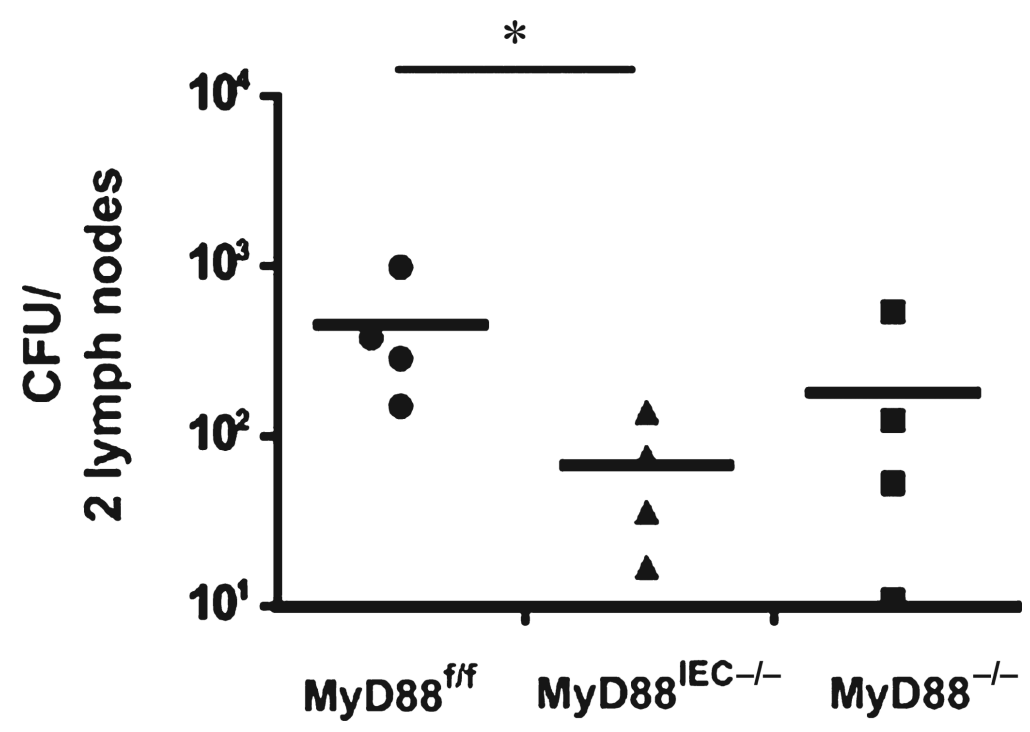

FIGURE 3.

I/R-exposed $M y d 88^{\text {IEC-/- }}$ mice display diminished bacterial translocation compared to $M y d 88^{\mathrm{f} / \mathrm{f}}$ mice. $M y d 88^{\mathrm{f} / \mathrm{f}}, M y d 88^{\mathrm{IEC}-/-}$, and $M y d 88^{-/-}$mice $(\mathrm{n}=4)$ were subjected to 60minute ischemia and 240-minute reperfusion. Two mesenteric lymph nodes adjacent to the affected ileal tissue were collected, weighed, homogenized, plated on brain heart infusion plates, and cultured under aerobic and anaerobic conditions for 24 hours. Colony-forming units were counted, and combined colony-forming units for aerobic and anaerobic conditions are shown. All graphs depict mean \pm SEM. $* P<0.05$. Results are representative of 2 independent experiments. 


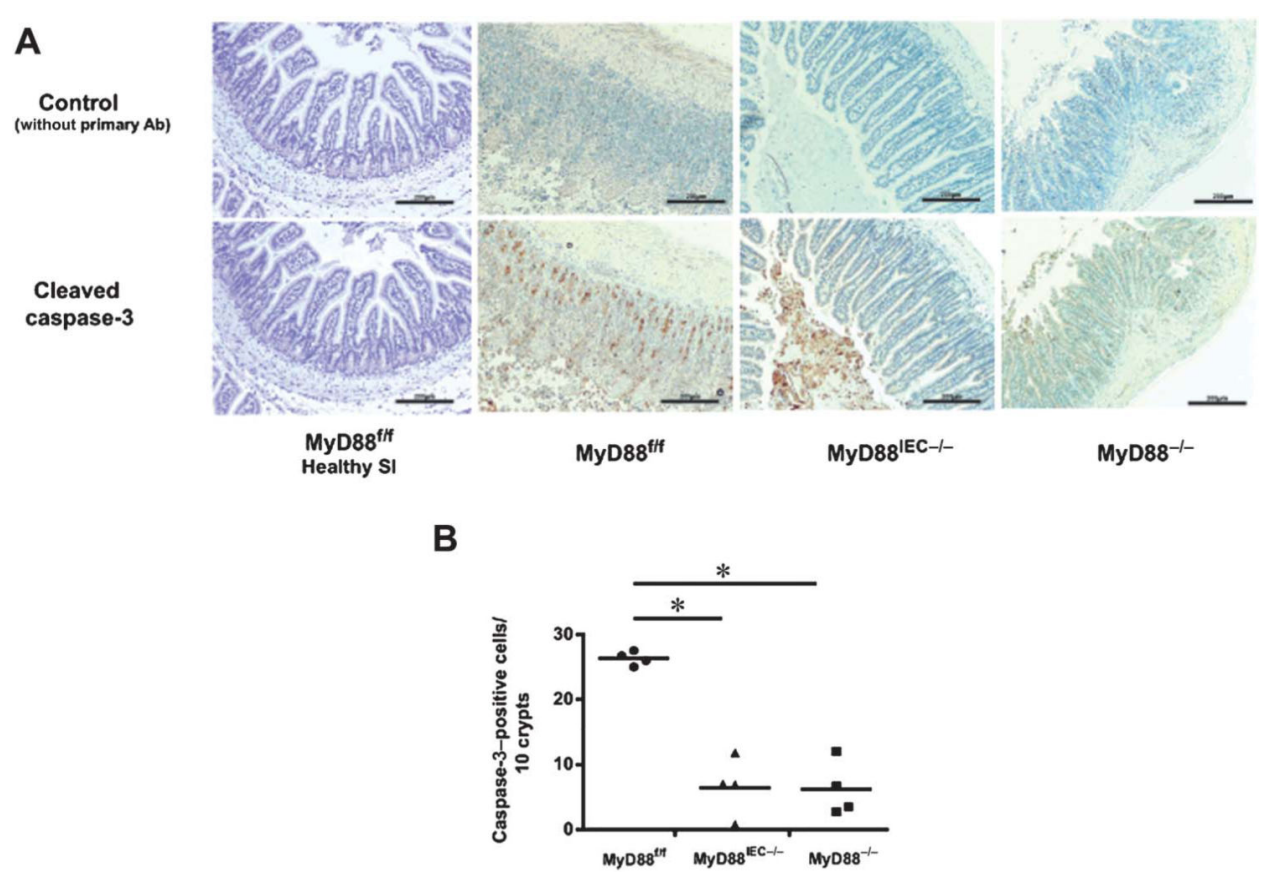

FIGURE 4.

I/R-exposed $M y d 88^{\mathrm{IEC}-/-}$ mice showed reduced apoptosis compared with $M y d 88^{\mathrm{f} / \mathrm{f}}$ mice. $M y d 88^{\mathrm{f} / \mathrm{f}}, M y d 88^{\mathrm{IEC}-{ }^{-}}$, and $M y d 88^{-/-}$mice $(\mathrm{n}=4)$ were subjected to 60 -minute ischemia and 90-minute reperfusion. A, IHC showing active caspase-3 in ileal tissues. Representative images are shown of 3 independent experiments. Scale bars $=200 \mu \mathrm{m}$. B, Average caspase-3-positive cells from ileal tissues of I/R-exposed $M y d 88^{\mathrm{f} / \mathrm{f}}$, Myd88 $\mathrm{IEC}-/-$, and Myd $88^{-/-}$mice. Data represent mean \pm SEM $P<0.01$. Results are representative of 2 independent experiments. 

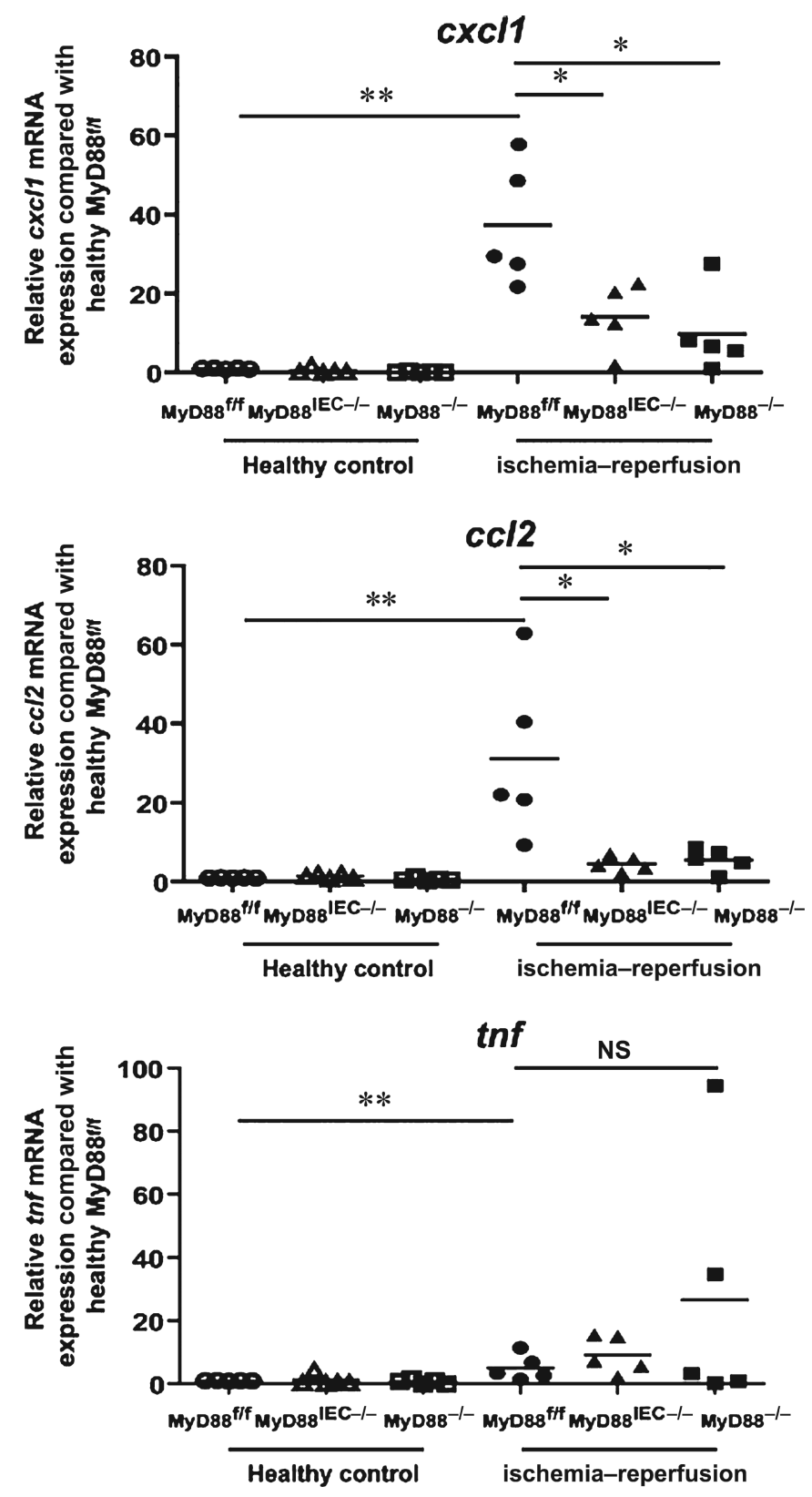

FIGURE 5.

I/R-exposed $M y d 88^{\mathrm{IEC}-/-}$ mice display lower chemokine mRNA expression. $M y d 88^{\mathrm{f} / \mathrm{f}}$,

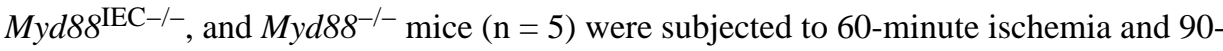
minute reperfusion and RT-PCR was performed on ileal tissue for $c c l 2$, cxcll, and tnf. Expression was normalized using gapdh. Fold expression is shown from I/R injured tissues compared with healthy control tissues. Data represent mean $\pm \mathrm{SEM}$; ${ }^{*} P<0.05$; $* * P<0.01$. Results are representative of 2 independent experiments. NS, not significant. 


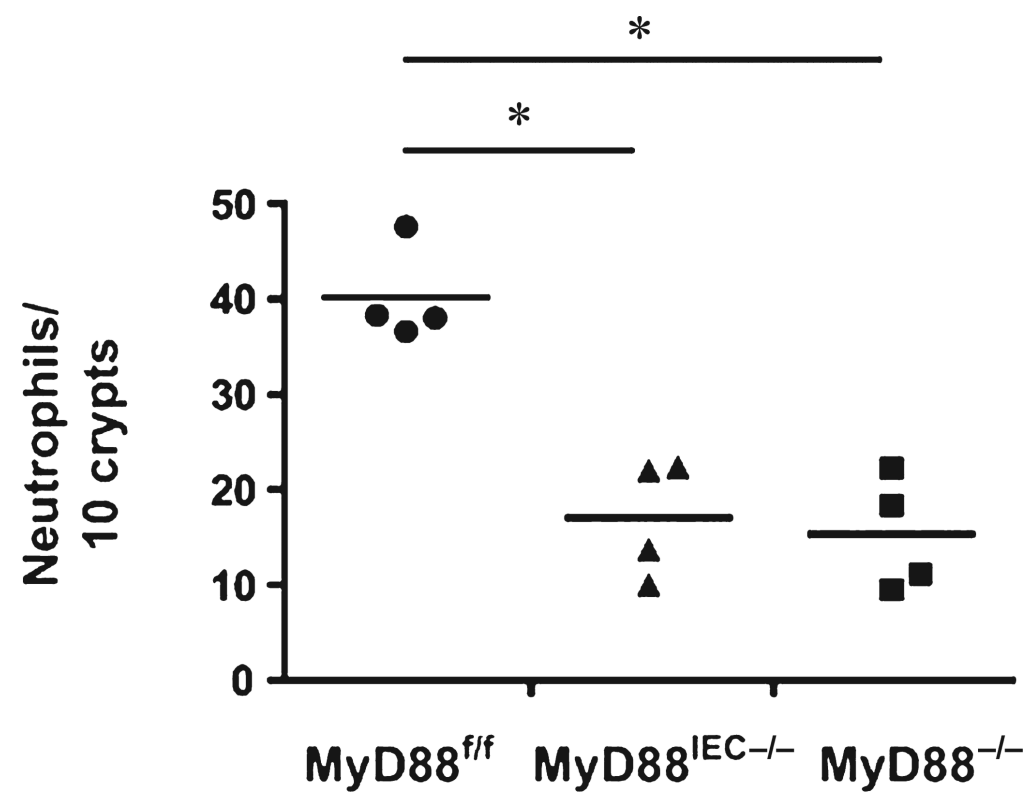

FIGURE 6.

I/R-exposed $M y d 88^{\mathrm{IEC}-/-}$ and $M y d 88^{-/-}$mice display impaired neutrophil influx compared with MyD88 8 f/f mice. $M y d 88^{\mathrm{f} / \mathrm{f}}, M y d 88^{\mathrm{IEC}-/-}$, and $M y d 88^{-/-}$mice $(\mathrm{n}=4)$ were subjected to 60-minute ischemia and 90-minute reperfusion, and myeloperoxidase expression was evaluated using IHC, and positive cells were counted. Data represent mean \pm SEM; * $P<$ 0.05 . Results are representative of 2 independent experiments. 


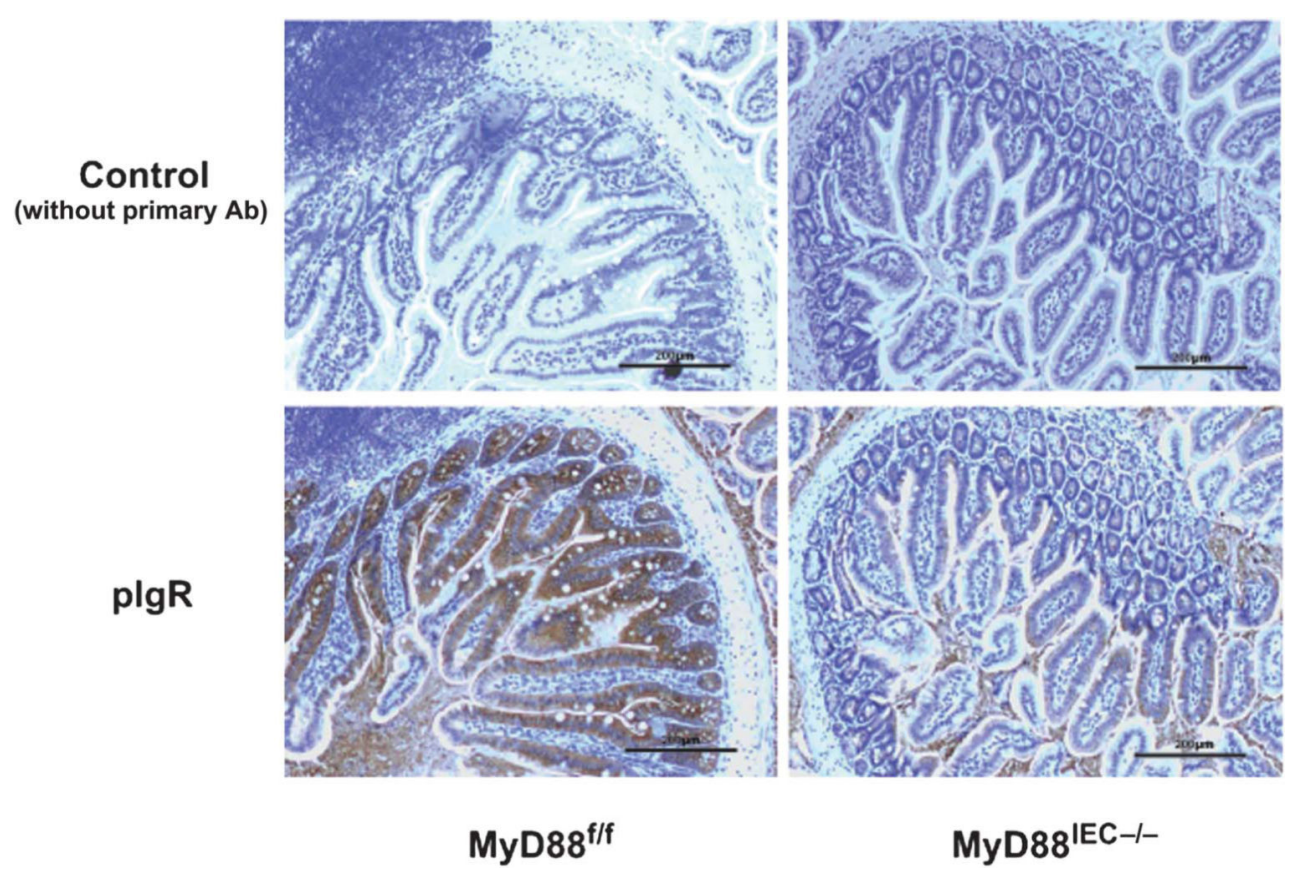

FIGURE 7.

Expression of the IgA transporter polymeric immunoglobulin receptor is decreased in Myd $88^{\mathrm{IEC}-/-}$ compared with $M y d 88^{\mathrm{f} / \mathrm{f}}$ mice. $M y d 88^{\mathrm{f} / \mathrm{f}}$ and $M y d 88^{\mathrm{IEC}-/-}$ mice $(\mathrm{n}=5)$ were euthanized, and polymeric immunoglobulin receptor expression was evaluated using IHC on healthy small intestinal tissue. Representative images are shown of 2 independent experiments. Scale bars $=200 \mu \mathrm{m}$. 


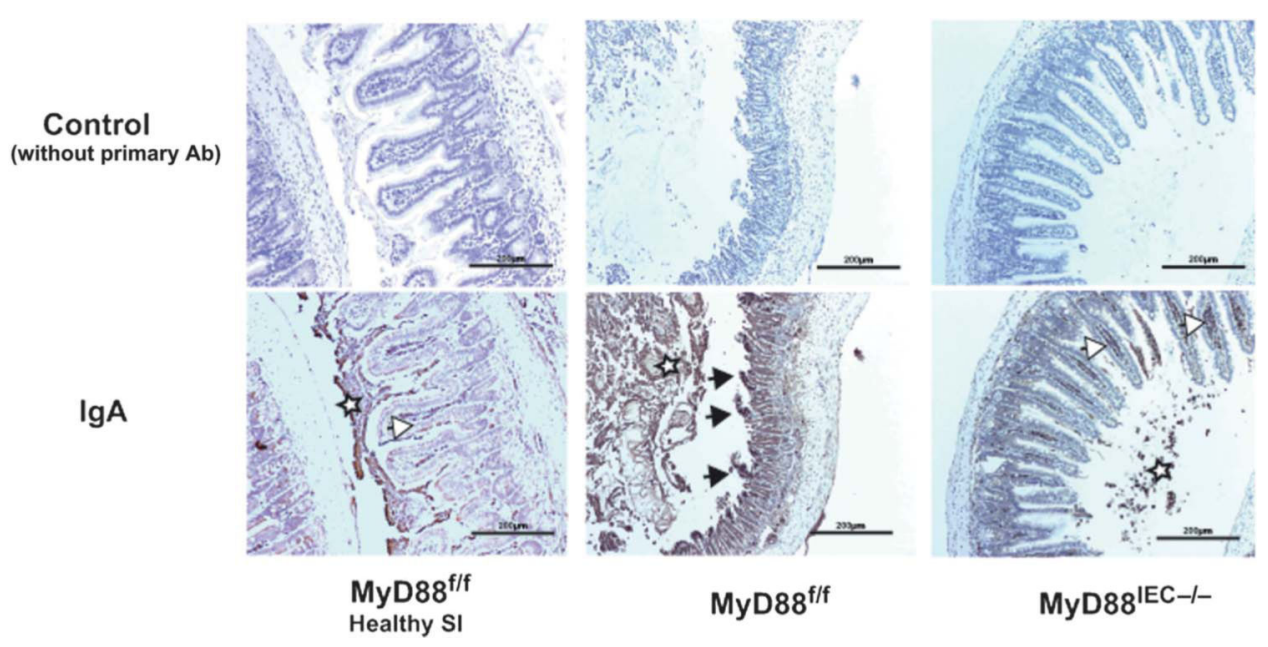

FIGURE 8.

IgA bound to ischemic tissue was reduced in I/R-exposed Myd88 IEC-I- compared with $M y d 88^{f / f}$ mice. $M y d 88^{f / f}$ and $M y d 88^{\mathrm{IEC}-/-}$ mice $(\mathrm{n}=5)$ were subjected to 60-minute ischemia only, and IgA expression was evaluated using IHC. IgA staining is also shown for healthy small intestinal tissue of $M y d 88^{\mathrm{f} / \mathrm{f}}$ mice (healthy SI). Black arrows: accumulation of IgA staining at the tip of the villi (region i.e., being affected first during I/R injury). White arrows: $\operatorname{Ig}$ A staining within B cells. White stars: staining of $\operatorname{IgA}$ within luminal debris. Scale bars $=200 \mu \mathrm{m}$. Representative images are shown of 2 independent experiments. 


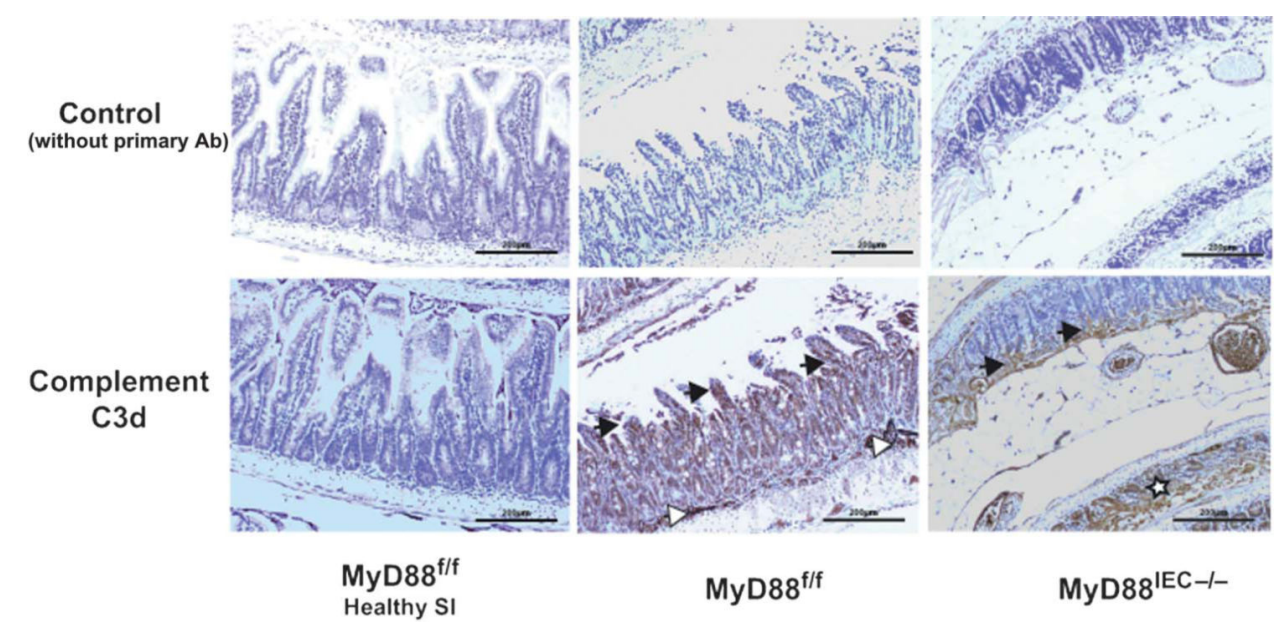

FIGURE 9.

Complement activation is impaired in I/R-exposed $M y d 88^{\mathrm{IEC}-/-}$ compared with $M y d 88^{\mathrm{f} / \mathrm{f}}$ mice. $M y d 88^{\mathrm{f} / \mathrm{f}}$ and $M y d 88^{\mathrm{IEC}-/-}$ mice $(\mathrm{n}=5)$ were subjected to 60-minute ischemia only, and complement $\mathrm{c} 3 \mathrm{~d}$ expression was determined using IHC. Complement $3 \mathrm{~d}$ staining is also shown for healthy small intestinal tissue of $M y d 88^{\mathrm{f} / \mathrm{f}}$ mice (healthy SI). Black arrows: accumulation of complement $3 \mathrm{~d}$ staining at the tip of the villi (region i.e., being affected first during I/R injury and region with accumulation of $\operatorname{IgA}$ ). White arrows: complement staining in submucosal blood vessels. White stars: complement staining only seen in crypts of Myd $88^{\text {IEC-l- }}$ mice when histological damage exceeds stage 2 (absence of villi but visible crypts). Scale bars $=200 \mu \mathrm{m}$. Representative images are shown of 2 independent experiments. 